EPJ Web of Conferences 66, 09008 (2014)

DOI: 10.1051/epjconf/ 20146609008

(C) Owned by the authors, published by EDP Sciences, 2014

\title{
A search for the $K^{-} p p$ bound state in the ${ }^{3} \mathrm{He}\left(K_{\text {in-flight }}^{-}, n\right)$ reaction at J-PARC
}

T. Hashimoto ${ }^{1, a}$, S. Ajimura ${ }^{2}$, G. Beer ${ }^{3}$, H. Bhang ${ }^{4}$, M. Bragadireanu ${ }^{5}$, P. Buehler ${ }^{6}$, L. Busso ${ }^{7,8}$, M. Cargnelli ${ }^{6}$, S. Choi ${ }^{4}$, C. Curceanu ${ }^{9}$, S. Enomoto ${ }^{2}$, D. Faso ${ }^{7,8}$, H. Fujioka ${ }^{11}$, Y. Fujiwara ${ }^{1}$, T. Fukuda ${ }^{12}$, C. Guaraldo ${ }^{9}$, R. S. Hayano ${ }^{1}$, T. Hiraiwa ${ }^{2}$, M. lio $^{14}$, M. Iliescu ${ }^{9}$, K. Inoue ${ }^{10}$, Y. Ishiguro ${ }^{11}$, T. Ishikawa ${ }^{1}$, S. Ishimoto ${ }^{14}$, T. Ishiwatari ${ }^{6}$, K. Itahashi ${ }^{13}$, M. Iwai ${ }^{14}$, M. Iwasaki ${ }^{15,13, b}$, Y. Kato ${ }^{13}$, S. Kawasaki ${ }^{10}$, P. Kienle ${ }^{16}$, H. Kou ${ }^{15}$, Y. Ma ${ }^{13}$, J. Marton ${ }^{6}$, Y. Matsuda ${ }^{17}$, Y. Mizoi ${ }^{12}$, O. Morra ${ }^{7}$, T. Nagae ${ }^{11}$, H. Noumi ${ }^{2}$, H. Ohnishi ${ }^{13}$, S. Okada ${ }^{13}$, H. Outa ${ }^{13}$, K. Piscicchia ${ }^{9}$, M. Poli Lener ${ }^{9}$, A. Romero Vidal ${ }^{9}$, Y. Sada ${ }^{11}$, A. Sakaguchi ${ }^{10}$, F. Sakuma ${ }^{13}$, M. Sato ${ }^{13}$, A. Scordo ${ }^{9}$, M. Sekimoto ${ }^{14}$, H. Shi ${ }^{1}$, D. Sirghi ${ }^{9,5}$, F. Sirghi ${ }^{9,5}$, K. Suzuki ${ }^{6}$, S. Suzuki ${ }^{14}$, T. Suzuki ${ }^{1}$, K. Tanida ${ }^{4}$, H. Tatsuno ${ }^{9}$, M. Tokuda ${ }^{15}$, D. Tomono ${ }^{13}$, A. Toyoda ${ }^{14}$, K. Tsukada ${ }^{18}$, O. Vazquez Doce ${ }^{9,19}$, E. Widmann ${ }^{6}$, B. K. Wuenschek ${ }^{6}$, T. Yamaga ${ }^{10}$, T. Yamazaki ${ }^{1,13}$, H. Yim ${ }^{20}$, Q. Zhang ${ }^{13}$, and J. Zmeskal ${ }^{6}$ (J-PARC E15 Collaboration)

${ }^{1}$ Department of Physics, The University of Tokyo, Tokyo, 113-0033, Japan

${ }^{2}$ Research Center for Nuclear Physics (RCNP), Osaka University, Osaka, 567-0047, Japan

${ }^{3}$ Department of Physics and Astronomy, University of Victoria, Victoria BC V8W 3P6, Canada

${ }^{4}$ Department of Physics, Seoul National University, Seoul, 151-742, South Korea

${ }^{5}$ National Institute of Physics and Nuclear Engineering - IFIN HH, Romania

${ }^{6}$ Stefan-Meyer-Institut für subatomare Physik, A-1090 Vienna, Austria

${ }^{7}$ INFN Sezione di Torino, Torino, Italy

${ }^{8}$ Dipartimento di Fisica Generale, Universita' di Torino, Torino, Italy

${ }^{9}$ Laboratori Nazionali di Frascati dell' INFN, I-00044 Frascati, Italy

${ }^{10}$ Department of Physics, Osaka University, Osaka, 560-0043, Japan

${ }^{11}$ Department of Physics, Kyoto University, Kyoto, 606-8502, Japan

${ }^{12}$ Laboratory of Physics, Osaka Electro-Communication University, Osaka, 572-8530, Japan

${ }^{13}$ RIKEN Nishina Center, RIKEN, Wako, 351-0198, Japan

${ }^{14}$ High Energy Accelerator Research Organization (KEK), Tsukuba, 305-0801, Japan

${ }^{15}$ Department of Physics, Tokyo Institute of Technology, Tokyo, 152-8551, Japan

${ }^{16}$ Technische Universität München, D-85748, Garching, Germany

${ }^{17}$ Graduate School of Arts and Sciences, The University of Tokyo, Tokyo, 153-8902, Japan

${ }^{18}$ Department of Physics, Tohoku University, Sendai, 980-8578, Japan

${ }^{19}$ Excellence Cluster Universe, Technische Universität München, D-85748, Garching, Germany

${ }^{20}$ Korea Institute of Radiological and Medical Sciences (KIRAMS), Seoul, 139-706, South Korea

\begin{abstract}
We have collected the first physics data of an experimental search for the simplest kaonic nuclear bound state, " $K^{-} p p$ ", by the ${ }^{3} \mathrm{He}\left(K^{-}, n\right)$ reaction at J-PARC. We confirmed that our spectrometer system works as designed and observed clear peak structure
\end{abstract}

\footnotetext{
${ }^{\mathrm{a}} \mathrm{e}$-mail: hashimoto@nucl.phys.s.u-tokyo.ac.jp

${ }^{\mathrm{b}}$ Spokesperson
}

This is an Open Access article distributed under the terms of the Creative Commons Attribution License 2.0, which permits unrestricted use, distribution, and reproduction in any medium, provided the original work is properly cited. 
composed of the quasi-elastic $K^{-“} n " \rightarrow K^{-} n$ and the charge-exchange $K^{-“} p$ " $\rightarrow \bar{K}^{0} n$ reactions in the forward neutron spectrum.

\section{Introduction}

Recent theoretical studies show that an anti-kaon can be bound in nuclei to form so-called kaonic nuclei as a result of strongly attractive $\bar{K} N$ interaction in I $=0$ channels [1]. Among such a new form of nuclear matter, " $K^{-} p p$ " [2] is now attracting great interest as the simplest one. Many theoretical calculations have been progressed for the $K^{-} p p$ system, resulting in various binding energy and width predictions. Experimentally, however, only a small amount of information is available [3][4], which is not sufficient to discriminate between a variety of conflicting interpretations.

In this situation, we are carrying out an experimental search for the $K^{-} p p$ bound state at J-PARC (J-PARC E15 [5]). The most important key of our experiment is the $\left(K^{-}, n\right)$ reaction at $1 \mathrm{GeV} / \mathrm{c}$. In this reaction, neutron backgrounds from non-mesonic two-nucleon absorptions or hyperon decays are expected to be substantially suppressed and kinematically separated. In addition, by using a liquid ${ }^{3} \mathrm{He}$ target and a large acceptance detector surrounding it, we can detect decay particles from " $K^{-} p p^{\text {" }}$ to fully reconstruct the reaction kinematics.

In this paper, we describe our spectrometer system, its performance and a very preliminary result of the forward neutron spectrum obtained in our first physics data taking.

\section{The K1.8BR spectrometer system at J-PARC}

A new spectrometer system has been designed and constructed at the secondary beam line K1.8BR in the hadron hall of J-PARC [6]. Figure 1 shows the schematic view of the spectrometer system.

\subsection{K1.8BR beam line and beam spectrometer}

The K1.8BR beam line delivers secondary beams of charged particles with momenta up to $1.2 \mathrm{GeV} / \mathrm{c}$, which are purified by an electro static separator in combination with two correction magnets and two vertical slits. Kaons are identified by using an Aerogel Cherenkov counter at a trigger level. The momentum of the secondary beam is analyzed by a beam line spectrometer with a momentum resolution of $2.2 \times 10^{-4}$ at $1 \mathrm{GeV} / \mathrm{c}$. The measured $1 \mathrm{GeV} / \mathrm{c}$ kaon yield at $24 \mathrm{~kW}^{1}$ primary beam power was $1.5 \times 10^{4}$ per spill with a $K^{-} / \pi^{-}$ratio of 0.45 .

\subsection{Cylindrical detector system}

Figure 2 shows a cylindrical detector system (CDS), which mainly consists of a solenoid magnet operated at $0.7 \mathrm{~T}$, a 15-layer cylindrical drift chamber (CDC) and a 36-segmented cylindrical detector hodoscope $(\mathrm{CDH})$. The CDS covers $60 \%$ of solid angle from the target and has good capability of particle identification as shown in Fig. 3. The $K_{s}^{0} \rightarrow \pi^{+} \pi^{-}$(Fig. 4) and $\Lambda \rightarrow p \pi^{-}$decays are successfully reconstructed with the designed performance, which corresponds to $10 \mathrm{MeV} / c^{2}$ invariant mass resolution for the expected " $K^{-} p p$ " $\rightarrow \Lambda p \rightarrow \pi^{-} p p$ decay channel.

\subsection{Forward counters}

One of the most unique features of our spectrometer system is a large-acceptance high-resolution neutron counter (NC) with a flight length of $\sim 15 \mathrm{~m}$. The geometrical acceptance is $\sim 20 \mathrm{msr}$ and the detection efficiency for a $1 \mathrm{GeV} / \mathrm{c}$ neutron is estimated to be $\sim 35 \%$. A beam sweeping magnet and two sets of veto counter arrays remove charged particles from the NC. We also measure the ${ }^{3} \mathrm{He}\left(K^{-}, p\right)$ reactions with a proton counter array to investigate the isospin dependence of the $\bar{K} N$ interaction.

\footnotetext{
${ }^{1} 3.0 \times 10^{13}$ protons per pulse at $30 \mathrm{GeV} / \mathrm{c}, 6$ second repetition cycle
} 


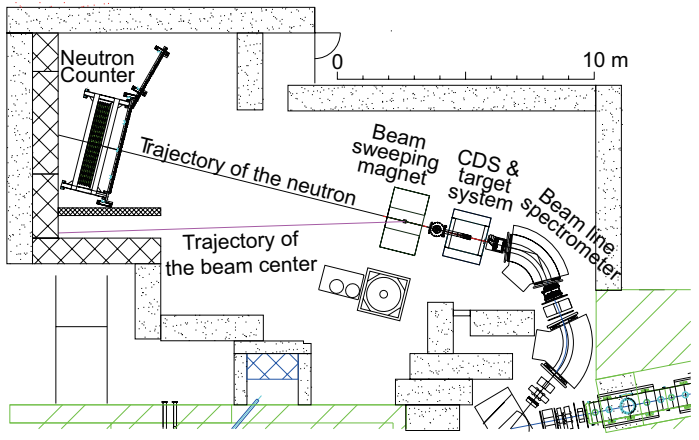

Figure 1. Schematic view of the J-PARC K1.8BR spectrometer [6].

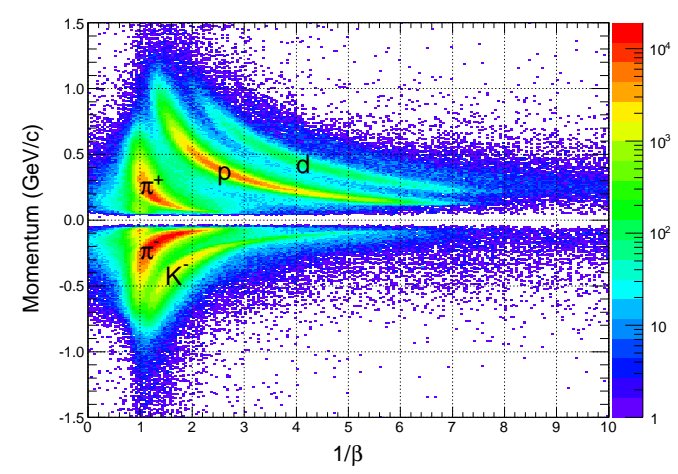

Figure 3. Performance of particle identification by the CDS. $1 / \beta$ was measured by the TOF from the reaction point to $\mathrm{CDH}$, and the momentum was obtained by the curvature of the $\mathrm{CDC}$ track.

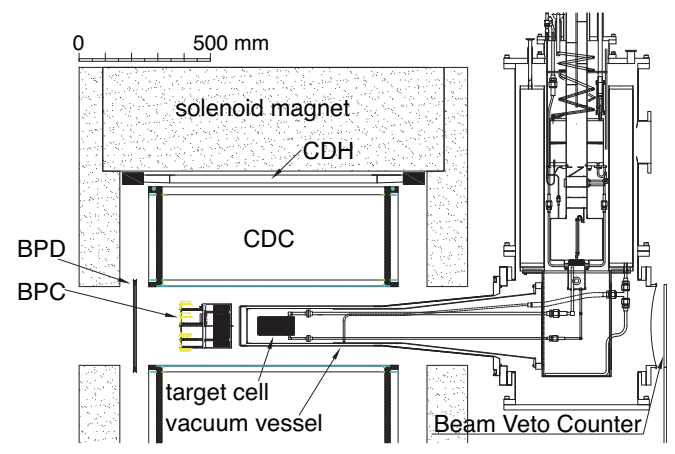

Figure 2. Schematic drawing of the cylindrical detector system with a liquid helium-3 target system [6].

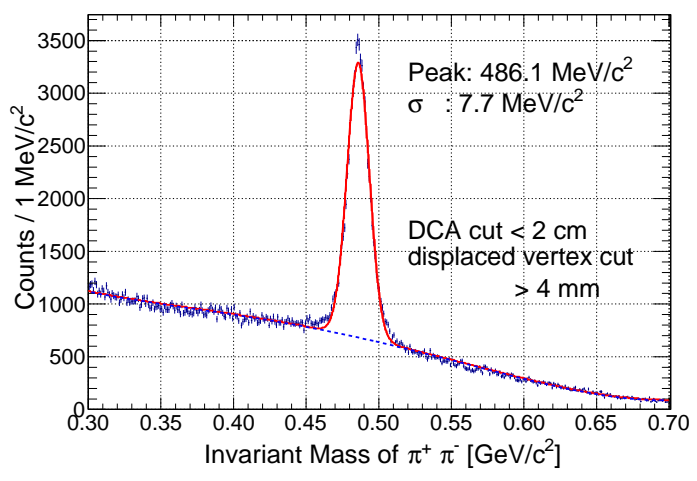

Figure 4. Invariant mass distribution of $\pi^{+} \pi^{-}$pairs reconstructed by the CDS. The spectrum is fitted with a Gaussian and a background curve. The centroid and resolution of $K_{s}^{0}$ are well reproduced by a detailed detector simulation.

\section{E15 first physics run}

The first physics run of the J-PARC E15 experiment was carried out in March and May, 2013. About $1 \%$ statistics of the full proposal was collected in this period with an integrated beam power of $15 \mathrm{~kW}$ $\times$ week, which corresponds to $5 \times 10^{9}$ kaons on the helium- 3 target.

\subsection{Neutron spectrum at forward angle}

Figure 5 and Fig. 6 show the $1 / \beta$ spectrum of neutral particles and the neutron missing mass spectrum measured by the NC with the data took in May ( $\sim 80 \%$ of the full statistics ), respectively. In both spectra, we requested at least one charged track in the CDS to reconstruct the reaction vertex and then we required the vertex to be roughly in the helium-3 target region. The TOF resolution of the system is evaluated to be $\sim 180 \mathrm{ps}$ from the $\gamma$ peak in Fig. 5, which is equivalent to $\sim 10 \mathrm{MeV} / \mathrm{c}^{2}$ missing mass resolution for the neutrons in the region of interest ( neutron momentum around $1.2 \mathrm{GeV} / \mathrm{c}$ ). A clear gap between the $\gamma$ peak width and the broad neutron distribution is showing that the accidental background is very small, even without any optimization of offline threshold for the energy deposit 


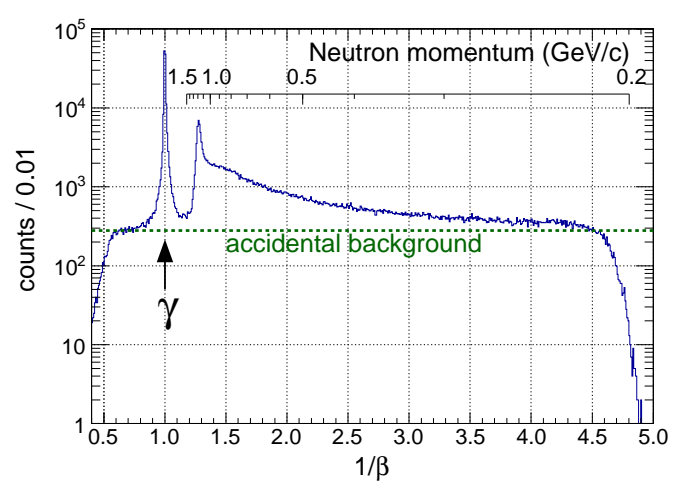

Figure 5. $1 / \beta$ spectrum of neutral particles, obtained by TOF analysis using a time-zero counter (T0) and the NC. The dotted line shows an accidental background contribution estimated from the left shoulder of the $\gamma$ peak.

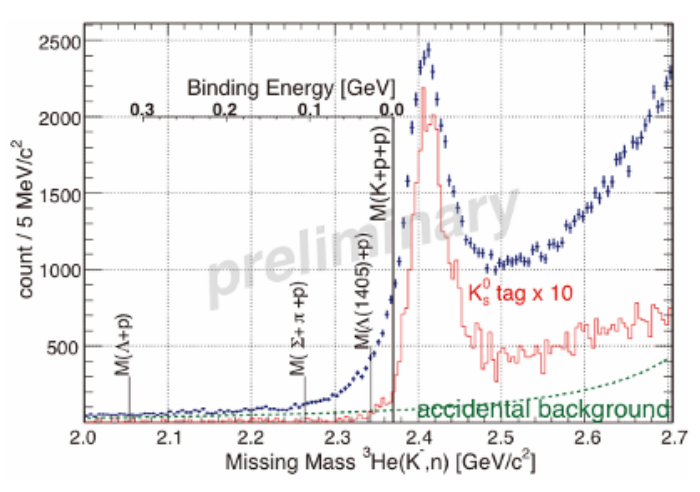

Figure 6. Missing mass spectrum of neutrons at forward angle. At least one charged track in CDS is requested to reconstruct the reaction vertex. A spectrum with $K_{s}^{0}$ tag by the CDS is overlaid with a scale factor of 10 . The dotted line shows the accidental background obtained in Fig. 5. The " $K^{-} p p$ " binding threshold is indicated by a solid line.

on NC. In Fig. 6, a peak structure due to the quasi-elastic $K^{-“} n " \rightarrow K^{-} n$ and the charge-exchange $K^{-“} p$ " $\rightarrow \bar{K}^{0} n$ reactions is seen just above the " $K^{-} p p$ " binding threshold $\left(2.37 \mathrm{GeV} / c^{2}\right)$. From the $K_{s}^{0}$ tagged spectrum overlaid in Fig. 6, the accuracy of the missing mass scale in the region of interest is evaluated to be better than $10 \mathrm{MeV} / \mathrm{c}^{2}$ at the current analysis stage.

\section{Summary and Outlook}

We constructed a new spectrometer system at K1.8BR beam line in the hadron hall of J-PARC and performed the first physics data taking with the ${ }^{3} \mathrm{He}\left(K^{-}, n\right)$ reaction at $1 \mathrm{GeV} / \mathrm{c}$ to search for the simplest kaonic nuclear bound state, " $K^{-} p p$ ". $5 \times 10^{9}$ kaons were irradiated on the helium-3 target and $3 \times 10^{5}$ forward neutrons were recorded. We confirmed that all components of the spectrometer system worked fine and the clear peak of the quasi-elastic and the charge-exchange reactions were observed in the neutron spectrum at forward angle.

Further analysis is now under way. For the neutron spectrum, we will convert vertical axis to the absolute cross section, reduce background events by optimizing various cut conditions and decompose the spectrum by tagging decay particles in the CDS. An exclusive study of $\Lambda p n$ final state is one of the most important subjects. The forward proton channel and hyperon productions are also our interests. These results will appear soon.

\section{References}

[1] Y. Akaishi and T. Yamazaki, Phys. Rev. C65 (2002) 044005.

[2] T. Yamazaki and Y. Akaishi, Phys. Lett. B535 (2002) 70.

[3] M. Agnello, G. Beer, L. Benussi et al., Phys. Rev. Lett. 94 (2005) 212303.

[4] T. Yamazaki, M. Maggiora, P. Kienle et al., Phys. Rev. Lett. 104 (2010) 132502.

[5] M. Iwasaki et al., J-PARC E15 proposal, http://j-parc.jp/NuclPart/pac_0606/pdf/p15-Iwasaki.pdf.

[6] K. Agari et al., Prog. Theor. Exp. Phys. (2012) 02B011. 